\title{
Hemelipoglycoprotein from the ornate sheep tick, Dermacentor marginatus: structural and functional characterization
}

\author{
Jarmila Dupejova ${ }^{1}$, Jan Sterba ${ }^{1,2^{*}}$, Marie Vancova ${ }^{1,2}$, Libor Grubhoffer ${ }^{1,2^{*}}$
}

\begin{abstract}
Background: Tick carrier proteins are able to bind, transport, and store host-blood heme, and thus they function also as antioxidants. Nevertheless, the role of carrier proteins in ticks is not fully understood. Some of them are found also in tick males which do not feed on hosts to such an extent such as females (there are differences in male feeding in different tick species) and thus they are not dealing with such an excess of heme; some of the carrier proteins were found in salivary glands where the processing of blood and thus release of heme does not occur. Besides, the carrier proteins bind relatively low amounts of heme (in one case only two molecules of heme per protein) compared to their sizes (above $200 \mathrm{kDa}$ ).

The main aim of this study is the biochemical characterization of a carrier protein from the ornate sheep tick Dermacentor marginatus, hemelipoglycoprotein, with emphasis on its size in native conditions, its glycosylation and identification of its modifying glycans, and examining its carbohydrate-binding specificity.
\end{abstract}

Results: Hemelipoglycoprotein from D. marginatus plasma was purified in native state by immunoprecipitation and denatured using electroelution from SDS-PAGE separated plasma. The protein (290 kDa) contains two subunits with molecular weights 100 and $95 \mathrm{kDa}$. It is glycosylated by high-mannose and complex N-glycans HexNAc${ }_{2} \mathrm{Hex}_{9}$, $\mathrm{HexNAc}_{2} \mathrm{Hex}_{10}, \mathrm{HexNAc}_{4} \mathrm{Hex}_{7}$, and HexNAc $\mathrm{Hex}_{8}$. The purified protein is able to agglutinate red blood cells and has galactose- and mannose-binding specificity. The protein is recognized by antibodies directed against plasma proteins with hemagglutination activity and against fibrinogen-related lectin Dorin M from the tick Ornithodoros moubata.

It forms high-molecular weight complexes with putative fibrinogen-related proteins and other unknown proteins under native conditions in tick plasma. Feeding does not increase its amounts in male plasma. The hemelipoglycoprotein was detected also in hemocytes, salivary glands, and gut. In salivary glands, the protein was present in both glycosylated and nonglycosylated forms.

Conclusion: A $290 \mathrm{kDa}$ hemelipoglycoprotein from the tick Dermacentor marginatus, was characterized. The protein has two subunits with 95 and $100 \mathrm{kDa}$, and bears high-mannose and complex N-linked glycans. In hemolymph, it is present in complexes with putative fibrinogen-related proteins. This, together with its carbohydrate-binding activity, suggests its possible involvement in tick innate immunity. In fed female salivary glands, it was found also in a form corresponding to the deglycosylated protein.

\section{Background}

Ticks are obligate ectoparasites of mammals, birds, and reptiles which feed only on blood of their hosts. Blood provides a rich source of nutrients needed for processes

\footnotetext{
*Correspondence: sterbaj@paru.cas.cz; liborex@paru.cas.cz

${ }^{1}$ Faculty of Science, University of South Bohemia, Branisovska 31, CZ-37005 Ceske Budejovice, Czech Republic

Full list of author information is available at the end of the article
}

associated with development to the next life-stage and egg production. During the digestion of the blood, erythrocytes are lysed and heme is released. As ticks do not possess the ability to synthesize heme [1], they utilize the host-blood heme. For this reason, as well as to neutralize the toxic effects of free heme, ticks use carrier proteins which bind and store it. Their nomenclature is

\section{Biomed Central}


not uniform and the proteins are called different names; some of them are described below.

Hemelipoglyco-carrier proteins (CP) are the most abundant proteins in the hemolymph $[2,3]$. The best studied are the CPs in hard ticks, Dermacentor variabilis [4] and Rhipicephalus microplus [2]. Hemelipoprotein (HeLp), carrier protein isolated from $R$. microplus, has molecular weight of about $354 \mathrm{kDa}$ and consists of two subunits with $103 \mathrm{kDa}$ and $92 \mathrm{kDa}$. It occurs mainly in hemolymph of adult tick stages in concentrations of around $50 \mathrm{mg} / \mathrm{ml}$ and is one of the most abundant hemolymph proteins. This molecule is able to bind heme in the ratio of two moles of heme to one mole of native HeLp and contains 3\% carbohydrates, and 33\% lipids [2]. HeLp carbohydrates contain mainly mannose which comprises more than $90 \%$ of all carbohydrates present in HeLp which corresponds to glycans found in ticks [[5], unpublished results]. The protein contains also neutral lipids, phospholipids, cholesterol esters, and cholesterol oleate. Labeling of HeLp with ${ }^{55} \mathrm{Fe}$ showed that this protein participates in heme transportation from hemolymph into ovaries during oogenesis [2].

Another $\mathrm{CP}$, hemelipoglyco-carrier protein (DvCP), found in hemolymph of both male and female ticks $D$. variabilis, shows a significant sequence homology with HeLp [4-7]. DvCP has a molecular weight of $210 \mathrm{kDa}$ and has two subunits with molecular weight of $98 \mathrm{kDa}$ and $92 \mathrm{kDa}$. Similarly to HeLp, DvCP contains lipids and carbohydrates [4]. DvCP was localized to fat body, salivary glands, ovary, and muscles of partially fed females [3].

The role of carrier-proteins in ticks is not fully understood. Some of them are found also in males which engorge a more limited volume of blood and thus are dealing with lower amounts of heme [4]. Moreover, the carrier protein persisted in $D$. variabilis males hemolymph in higher concentrations and for a longer period of time after detachment as compared to tick females [4]. Thus, it is possible that these proteins play also roles other than transport and storage of the heme.

In the current study, hemelipoglycoprotein (HLGP) from the hemolymph of the tick $D$. marginatus, closely related to DvCP, was isolated and characterized by electrophoretic and blotting techniques, surface plasmon resonance (SPR) and MALDI-TOF/TOF (Matrix-assisted laser desorption/ionization-Time-of-flight/Time-offlight) and ESI-FT-ICR (Electrospray ionization-Fouriertransform ion cyclotron resonance) mass spectrometry analysis. The molecular weight of the protein and its subunits, $N$-linked glycans modifying the protein, and carbohydrate-binding specificity were determined. The protein was also recognized by antibodies directed against Dorin $M$, a fibrinogen-related lectin from Ornithodoros moubata [8], and sera raised against hemagglutination activity of the $D$. marginatus hemolymph.

\section{Methods}

\section{Ticks}

Unfed and partially-fed (further referred to as "unfed" and "fed") females and males of the tick Dermacentor marginatus were obtained from the tick facility of the Institute of Parasitology, Biology Centre of the Academy of Sciences of the Czech Republic in České Budějovice. After metamorphosis, males and females were separated and kept in glass vials in wet chambers at $26^{\circ} \mathrm{C}$ until feeding/plasma and tissue preparation. Females and males were allowed to feed on laboratory guinea pigs for 6 days.

\section{Plasma and tissue preparation}

Hemolymph was collected after cutting off a part of tick's anterior leg with fine scissors. The hemolymph from 8 to 10 ticks (approx. 10-15 $\mu \mathrm{l}$ and $5 \mu \mathrm{l}$ for unfed and fed ticks, respectively) was collected directly into 50 $\mu \mathrm{l}$ of $0.9 \% \mathrm{NaCl}$ containing protease inhibitors (Pierce, Thermo Fisher, Rockford, IL). The solution was centrifuged at $4^{\circ} \mathrm{C}$ for $10 \mathrm{~min}$ at $100 \mathrm{~g}$ to pellet the hemocytes. The resulting supernatant was then clarified at $23000 \mathrm{~g}$ for $20 \mathrm{~min}$ and both the plasma and hemocyte fractions were stored at $-20^{\circ} \mathrm{C}$.

Gut and salivary glands were dissected from partiallyfed females, thoroughly washed in phosphate-buffered saline, $\mathrm{pH}$ 7.4 (PBS) to remove possible contamination, and organs from five ticks were homogenized with 300 $\mu \mathrm{l}$ PBS for 2 minutes at frequency of $30 \mathrm{~Hz}$ in TissueLyser II (Qiagen, Hombrechtikon, Switzerland) and stored at $-70^{\circ} \mathrm{C}$.

\section{SDS-PAGE and Blue Native/SDS-PAGE electrophoresis}

For SDS-PAGE, plasma samples were diluted 1:5 in PBS, mixed with loading buffer with or without reducing agent dithiothreitol (Fermentas, Thermo Fisher, Vilnius, Lithuania) and heated for $5 \mathrm{~min}$ at $95^{\circ} \mathrm{C}$. SDS-PAGE was performed on 4-17.5\% gradient gels [9] in MiniPROTEAN electrophoresis system (Bio-Rad, Hercules, CA). Gels were stained with PageBlue Protein Staining Solution (Fermentas). Approximately $5 \mu$ g proteins were loaded in the case of tick plasma and hemocytes, while $15 \mu \mathrm{g}$ of tick gut and salivary gland proteins were applied.

In the case of BN-PAGE/SDS-PAGE, samples were mixed with $5 \%$ glycerol and $0.01 \%$ Ponceau $S$ and separated on native gradient gel (3.5\% stacking, $6-13 \%$ separating gel) [10] together with native protein molecular weight standards (Sigma-Aldrich, St. Louis, MO) in Mini-PROTEAN electrophoresis system. Gels were stained with PageBlue Protein Staining Solution or used 
for second dimension of $2 \mathrm{D}$ electrophoresis. For the second dimension, the BN-PAGE separated proteins were used for Tris-Tricine SDS-PAGE [10]. Briefly, 12\% separating gel was prepared, gel strips from BN-PAGE were incubated for $1 \mathrm{~h}$ in cathode buffer $(0.1 \mathrm{M}$ Tris, $0.1 \mathrm{M}$ Tricine, $0.1 \%$ SDS, $\mathrm{pH}$ 8.25), inserted above the separating gel and overlaid with $4 \%$ stacking gel. Electrophoresis was performed for about 2.5 hours at a current limit of $50 \mathrm{~mA}$ using anode buffer $(0.1 \mathrm{M}$ Tris, $0.0225 \mathrm{M} \mathrm{HCl}, \mathrm{pH} 8.9$ ) and cathode buffer [10]. The separated proteins were stained with PageBlue Protein Staining Solution.

\section{Electroelution of HLGP}

Electroelution was performed using ElutaTube ${ }^{\text {max }}$ Protein, DNA and RNA Extraction and Dialysis Kit (Fermentas). Bands corresponding to HLGP were cut out of the gel, placed into ElutaTube vial with electrophoresis running buffer (0.025 M Tris, $0.192 \mathrm{M}$ glycine, 0.1\% SDS), and placed in the supporting tray in the electrophoresis tank with running buffer. Electroelution ran for 3 hours at $100 \mathrm{~V}$. Samples were precipitated by four volumes of acetone, pelleted, the precipitated proteins were airdried, and dissolved in water with resulting concentration of approximately $0.5 \mathrm{mg} / \mu \mathrm{l}$.

\section{Anti-HLGP and anti-HA polyclonal serum preparation}

Mice were housed in the Animal facility of the Institute of Parasitology, Biology Center of the ASCR in České Budějovice in plastic cages with sawdust bedding. Pellet diet and water were supplied ad libitum and mice were handled in accordance with the Animal Act of the Czech Parliament.

D. marginatus plasma proteins were separated by SDSPAGE and stained with PageBlue Protein Staining Solution (Fermentas). HLGP bands (corresponding to approximately $50 \mu \mathrm{g}$ of protein) were cut out and homogenized with $240 \mu \mathrm{l}$ PBS $(1 \times)$. Incomplete Freund's adjuvant was added in a 1:1 ratio and $80 \mu \mathrm{l}$ of this solution was subcutaneously injected to BALB/c mice. Immunization was repeated $3 \times$ every 14 days by subcutaneous injection of 80 $\mu \mathrm{l}$ of this solution. Blood sera were collected 14 days after the last immunization. Sera were supplemented with glycerol (1:1), aliquoted and stored at $-20^{\circ} \mathrm{C}$.

Anti-hemagglutination activity (anti-HA) serum was prepared as described elsewhere [11]. $20 \mu \mathrm{l}$ of tick hemolymph was added to $50 \mu \mathrm{l}$ of $2 \%$ suspension of mouse red blood cells. After $1 \mathrm{~h}$ incubation, the hemagglutinated cells were washed, resuspended in $200 \mu \mathrm{l}$ of PBS, and $50 \mu \mathrm{l}$ of the mixture was injected into mice. The immunization was repeated three times every 14 days. Blood sera were collected 14 days after the last immunization. Sera were supplemented with glycerol (1:1), aliquoted and stored at $-20^{\circ} \mathrm{C}$.

\section{Immunoblotting}

The electrophoretically separated proteins were transferred to PVDF membrane [12] for 1 hour at $20 \mathrm{~V}$. The PVDF membrane was washed in PBS, cut into strips, and incubated for 1 hour in 5\% skim powdered milk in PBS. Strips were then incubated for 1 hour in mouse anti-HLGP or anti-HA serum, washed with PBS-Tween $20(0,05 \%$ Tween 20 in PBS) and incubated with goat anti-mouse antibody conjugated with alkaline phosphatase (Vector Laboratories, Burlingame, CA) in 5\% milk. After incubation, strips were washed with PBS-Tween 20 and PBS. Reaction was developed in alkaline phosphatase-staining solution (Vector Laboratories) and after the development of sufficient signal it was stopped by washing the strips several times in Milli-Q water.

\section{Schiff staining and lectinoblotting of HLGP}

Electroeluted HLGP was separated by SDS-PAGE and electroblotted to PVDF membrane. The presence of glycosylation was detected with DIG Glycan Detection Kit (Roche Applied Science, Mannheim, Germany) and the glycan types modifying the HLGP were identified using DIG Glycan Differentiation Kit (Roche Applied Science) utilizing lectins SNA, GNA, DSA, PNA, and MAA II, conjugated with digoxigenin.

Membrane strips for Schiff staining were washed with PBS, the glycoproteins were oxidized by $10 \mathrm{mM}$ sodium periodate in $0.1 \mathrm{M}$ sodium acetate buffer, $\mathrm{pH} 5.5$ at room temperature for 20 minutes, washed in PBS, and incubated with DIG-3-O-succinyl-e-aminocaproic acid hydrazide in sodium acetate buffer for 1 hour at room temperature. After three washes in Tris-buffered saline, pH 7.5 (TBS, $0.05 \mathrm{M}$ Tris, $0.15 \mathrm{M} \mathrm{NaCl}$ ) the strips were blocked in blocking solution (provided by the manufacturer and diluted 1:9 in TBS) for 1 hour at room temperature, washed in TBS, incubated with anti-DIG antibodies conjugated with alkaline phosphatase in the blocking solution for 1 hour at room temperature, washed with TBS, and finally, the color reaction was developed in BCIP/NBT solution in $0.1 \mathrm{M}$ Tris, $0.05 \mathrm{M}$ $\mathrm{MgCl}_{2}, 0.1 \mathrm{M} \mathrm{NaCl}, \mathrm{pH} 9.5$. The reaction was stopped by thorough washing in Milli-Q water.

For lectinoblotting, the membrane strips were washed in TBS and blocked in blocking solution for 1 hour at room temperature. Next, the strips were washed in TBS and in TBS supplemented with $1 \mathrm{mM} \mathrm{CaCl}_{2}, 1 \mathrm{mM}$ $\mathrm{MgCl}_{2}, 1 \mathrm{mM} \mathrm{MnCl}$, and incubated in the supplemented TBS with the individual DIG-conjugated lectins for minimum 1 hour at room temperature. The strips were washed again in TBS, incubated with anti-DIG antibodies conjugated with alkaline phosphatase in the blocking solution for 1 hour at room temperature, washed with TBS, and the color reaction was developed as described above. 


\section{Enzymatic deglycosylation}

Electroeluted HLGP was deglycosylated using glycosidases Endo H (New England Biolabs, Ipswich, MA) or PNGase F (New England Biolabs) and PNGase A (Roche Applied Science) under reducing and non-reducing conditions. For deglycosylation using PNGase A/F under reducing conditions, $68 \mu \mathrm{l}$ of electroeluted HLGP was mixed with $10 \mu \mathrm{l}$ denaturation buffer (5\% SDS, $0.4 \mathrm{M}$ DTT) and heated on $95^{\circ} \mathrm{C}$ for $10 \mathrm{~min}$. The solution was mixed with $10 \mu \mathrm{l} 0.5 \mathrm{M}$ sodium phosphate, $\mathrm{pH}$ 7.4, 10 $\mu \mathrm{l}$ Nonident P-40, $0.5 \mu \mathrm{l}$ PNGase A, $0.5 \mu \mathrm{l}$ PNGase F, and $1 \mu \mathrm{l}$ Milli-Q water.

For deglycosylation using Endo $\mathrm{H}$ under reducing conditions, $68 \mu \mathrm{l}$ of electroeluted HLGP was mixed with 10 $\mu \mathrm{l}$ denaturation buffer (5\% SDS, 0.4 M DTT) and heated on $95^{\circ} \mathrm{C}$ for $10 \mathrm{~min}$. The solution was mixed with $10 \mu \mathrm{l}$ $0.5 \mathrm{M}$ sodium citrate, $\mathrm{pH} 5.5,2 \mu \mathrm{l}$ Endo $\mathrm{H}$, and $10 \mu \mathrm{l}$ Milli-Q water. Deglycosylation under non-reducing conditions was performed similarly with the denaturation step to be omitted. Deglycosylation reactions were performed overnight at $37^{\circ} \mathrm{C}$. All reactions were performed in duplicates.

$N$-linked glycans from HLGP and D. marginatus hemolymph were prepared from reduced and alkylated electroeluted proteins, which were digested by trypsin (Roche Applied Science) in PBS, overnight at $37^{\circ} \mathrm{C}$. The peptides were purified using C18 spin columns (Harvard Apparatus, Holliston, MA), vacuum-dried and deglycosylated as described above using a mixture of PNGase A and PNGase $\mathrm{F}$ to ensure the release of all $\mathrm{N}$-glycans. The released glycans were purified using a combination of C18 and active-charcoal spin columns (Harvard Apparatus) [13]. $\mathrm{N}$-glycans preparation and their subsequent MS analysis were performed in duplicates.

\section{Solid-phase permethylation of $\mathrm{N}$-glycans for MS analysis}

PNGase A/F-released glycans were vacuum dried, resuspended in $5 \mu \mathrm{l}$ of water, and $70 \mu \mathrm{l}$ of dimethylformamide and $25 \mu$ l of methyl iodide were added. The mixture was applied onto $\mathrm{NaOH}$ beads (Sigma-Aldrich) in spin-columns (Harvard Apparatus), incubated for 15 minutes, and the columns were centrifuged for 1 minute at $1500 \times$ G. $25 \mu \mathrm{l}$ of methyl iodide was added to the solution, again applied onto $\mathrm{NaOH}$ beads, incubated for 15 minutes, and centrifuged [13]. Next, the beads were washed with acetonitrile, centrifuged, and the solutions were pooled. The permethylated glycans were recovered by liquid/liquid extraction with chloroform and washing with $0.5 \mathrm{M} \mathrm{NaCl}$ and HPLC-grade water. Finally, the glycans were vacuum dried.

\section{Immunoprecipitation}

$50 \mu \mathrm{l}$ of $D$. marginatus hemolymph plasma, diluted 1:5 in $0.9 \% \mathrm{NaCl}$, was mixed with $1 \mu \mathrm{l}$ of $1 \%$ Tween 20 in
PBS, $5 \mu \mathrm{l}$ of protease inhibitors (Pierce), and with $444 \mu \mathrm{l}$ of PBS. The mixture was incubated with $5 \mu \mathrm{l}$ of anti$\mathrm{HA} /$ anti-HLGP serum at $4^{\circ} \mathrm{C}$ overnight. $10 \mu \mathrm{l}$ of magnetic Dynabeads with Protein G (Invitrogen, Carlsbad, CA) were added into the solution and incubated $45 \mathrm{~min}$ at room temperature. The beads were then washed sixtimes in $0.01 \%$ Tween 20 in PBS. HLGP was eluted using elution buffer containing primary amines (MicroLink Protein Coupling Kit, Pierce). Immunoprecipitation experiments were performed three-times with similar results.

\section{Surface plasmon resonance (SPR)}

Quantitative measurement of interactions between HLGP and carbohydrates was performed on BiaCore 3000 instrument (GE Healthcare, Buckinghamshire, UK) (National Centre for Biomolecular Research, Michaela Wimmerová and Lenka Malinovská, Masaryk University, Brno, Czech Republic). A chip with immobilized monosaccharides ( $\alpha$-D-galactose on channel 1, $\alpha$-D-mannose/ $\alpha$-L-fucose on channel 2, $\alpha$-D-mannose on channel 3, and $\alpha$-L-fucose on channel 4) was used. $15 \mu \mathrm{l}$ of samples were injected on the chip and the response of individual channels was monitored at a flow-rate of $5 \mu \mathrm{l} /$ min. Dilutions of the samples as well as samples containing EDTA $(25 \mathrm{mM})$ were injected for complementary experiments. Both experiments with and without EDTA were performed in duplicates.

\section{Hemmaglutination assay}

The determination of hemagglutination activity (HA) was performed in 96-well U-shaped microtitration plates by serial two-fold dilution of $50 \mu \mathrm{l}$ samples in $50 \mu \mathrm{l}$ of $0.15 \mathrm{M} \mathrm{NaCl}$ [9]. Next, $50 \mu \mathrm{l}$ of $2 \%(\mathrm{v} / \mathrm{v})$ suspension of mouse erythrocytes in $0.15 \mathrm{M} \mathrm{NaCl}$ was added to each well. The titer of HA was evaluated after $1 \mathrm{~h}$ incubation at room temperature and expressed as the reciprocal value of the last sample dilution causing visible agglutination.

\section{Mass spectrometry}

Purified HLGP or gel slices containing HLGP were trypsinized according to the manufacturer's instructions (Roche Applied Science) and subjected to LC-MS analysis. Peptides were separated by HPLC on C18 silica (Agilent, Santa Clara, CA) using acetonitrile gradient (5$80 \%)$ as a mobile phase and analyzed by FT-ICR mass spectrometer (Thermo Fisher). The obtained data were compared to Acari non-redundant and Swiss-Prot databases using Mascot (a proprietary identification program, Matrix Science, Boston, MA) using strict criteria.

The permethylated glycans were spotted on matrixassisted laser desorption/ionization (MALDI) plate with 2,5-dihydroxybenzoic acid and analyzed on 4800 
MALDI TOF/TOF Analyzer (Applied Biosystems, Life Technologies, Carlsbad, CA) in positive-ion mode. The data were converted to mzXML format and further analyzed by mMass [14] and Glycoworkbench [15] software. Mass spectrometric analyses were performed in National Center for Glycomics and Glycoproteomics, Indiana University, Bloomington, IN, USA (Benjamin F. Mann, William R. Alley, Jr., and Milos V. Novotny).

\section{Results}

\section{HLGP detection and identification in D. marginatus hemolymph}

Putative lectin molecules in the hemolymph of the tick Dermacentor marginatus were detected using sera and antibodies recognizing similar molecules in tick species. The sera used were directed against HA of the hemolymph of $D$. marginatus tick in addition to antibodies recognizing Dorin $M$ protein, a lectin from the hemolymph of the tick Ornithodoros moubata. Several putative carbohydrate-binding proteins with molecular weight around $37 \mathrm{kDa}, 79 \mathrm{kDa}, 80 \mathrm{kDa}$, and a highmolecular protein with molecular weight of approximately $290 \mathrm{kDa}$ were identified in non-reduced hemolymph (Figure 1A). The reactivity of antibodies differed for these proteins and the $290 \mathrm{kDa}$ molecule was the only one, recognized by all the antibodies.

The bands containing all four proteins were excised from the SDS-PAGE gel and mass spectrometric analyzes (ESI-FT-ICR MS) were performed. Although the three low molecular weight proteins were not identified, the $290 \mathrm{kDa}$ protein (asterisk, Figure 1A) was identified as hemelipoglycoprotein (HLGP) related to hemelipoglyco-carrier protein $(\mathrm{CP})$ from $D$. variabilis. The amino acid sequence coverage was $10.2 \%$ and $4.0 \%$ (see Additional File 1; Table S1 and Additional File 2; Table S2, respectively) for hemelipoglycoprotein precursor 1 and 2 of the homologous HLGP from $D$. variabilis, respectively.

Next, polyclonal antibodies against this protein were raised. The following immunoblot analysis showed that the anti-HLGP antibodies recognized the $290 \mathrm{kDa}$ protein under non-reducing conditions and two subunits with molecular weights of 95 and $100 \mathrm{kDa}$ under reducing conditions (data not shown).

\section{Isolation of HLGP}

Further, HLGP was isolated from the plasma under both native and denaturing conditions. Native protein was purified using immunoprecipitation with Protein G coupled to magnetic beads and anti-HLGP serum (see Additional File 3; Figure S1; asterisk). The denatured protein was isolated from electrophoretically separated hemolymph by cutting out the HLGP protein from the gel and its subsequent electroelution (see Additional File
3; Figure S1; asterisk). In both cases, the purification of HLGP was successful.

\section{Characterization of HLGP}

To assess the molecular weight of the protein under native conditions, blue native-PAGE and subsequent SDS-PAGE of tick plasma were performed. The results show that HLGP (arrow, Figure 2A) is part of a highmolecular weight complex together with some other proteins which have not been identified yet (Figure 2A). Under native conditions, the complex has size of approximately $450 \mathrm{kDa}$ (arrow, Figure 2B). However, some of these proteins are recognized by antibodies directed against tick lectins and HA (data not shown).

Schiff staining of HLGP showed, that the protein is indeed glycosylated (Figure 3A, lane 2). The glycan moieties were further analyzed by lectin-affinity staining. Terminal mannose-specific GNA lectin and Gal- $\beta(1-4)-$ GlcNAc-specific DSA lectin showed strong binding to HLGP (Figure 3A, lanes 4,5), $\alpha(2-6)$-linked sialic acidspecific SNA lectin stained HLGP weakly (Figure 3A,

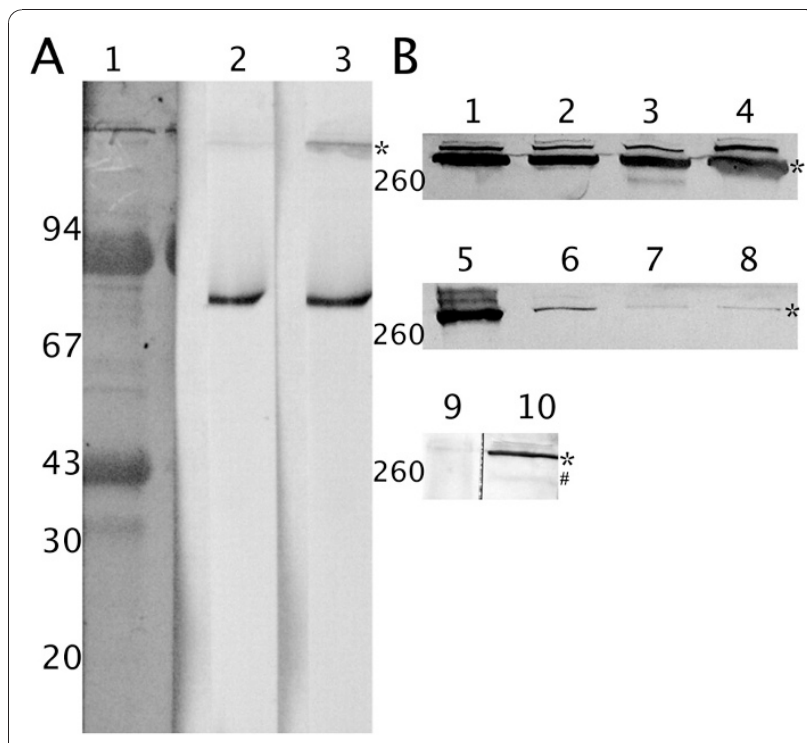

Figure 1 Identification of putative FReD proteins in $D$. marginatus plasma and detection of HLGP in tick tissues. Molecular weights of standard proteins are depicted. A) Immunoblotting of non-reduced electrophoretically separated $D$. marginatus plasma proteins. 1 - D. marginatus plasma proteins, stained with Coomassie Brilliant Blue, 2 - immunostaining of plasma proteins using antibodies against HA of $D$. marginatus hemolymph serum, 3 - immunostaining of plasma proteins by anti-Dorin M antibodies. B) Immunoblotting of HLGP in D. marginatus tissues using mouse polyclonal anti-HLGP serum. 1 - fed female plasma, 2 unfed female plasma, 3 - fed male plasma, 4 - unfed male plasma, 5 - fed female hemocytes, 6 - unfed female hemocytes, 7 - fed male hemocytes, 8 - unfed male hemocytes, 9 - fed female gut, 10 - fed female salivary glands. * marks the position of native glycosylated HLGP, \# marks the deglycosylated form of HLGP. 


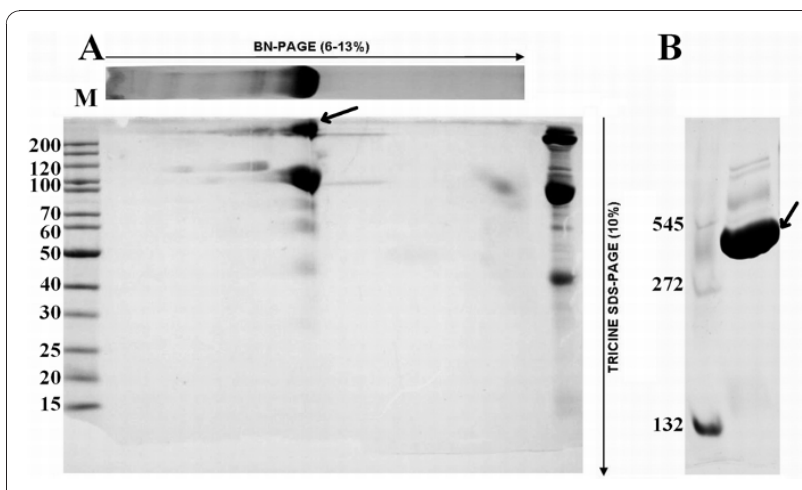

Figure $22 \mathrm{D}$ and native electrophoreses of plasma proteins and HLGP. A) Blue native/SDS-PAGE 2 D electrophoretic separation of $D$. marginatus plasma proteins, stained by Coomassie Brilliant Blue (CBB). BN-PAGE separated plasma proteins are showed above the gel and acrylamide gradients are depicted above the gel (native electrophoresis) and on the right side (SDS-PAGE electrophoresis). HLGP is marked with an arrow. B) Blue native PAGE of $D$.

marginatus plasma proteins, stained by CBB. 1 - native molecular weight standard, 2 - tick plasma. Complex of HLGP with other proteins is marked with an arrow.

lane 3), while Gal- $\beta(1-3)$-GalNAc and $\alpha(2-3)$-linked sialic acid-specific lectins PNA and MAA did not give positive reaction (data not shown). These results suggest the presence of $\mathrm{N}$-glycans of hybrid and complex type with terminal mannose, galactose or sialic acid. DSA lectin binding to HLGP can indicate the presence of $O$ glycans, too.

The proportion of the glycan part of the molecule was determined from deglycosylation reactions of HLGP. Enzymes Endo $\mathrm{H}$ and the mixture of PNGase A/F were used to cleave off the $N$-glycans. Anti-HLGP serum detected two proteins with $250 \mathrm{kDa}$ and $290 \mathrm{kDa}$ in both PNGase A/F (Figure 3B, lane 3) and Endo $\mathrm{H}$ treated purified native HLGP (Figure 3B, lane 4). As these endoglycosidases work under native conditions with a slower reaction rate, the larger protein (asterisks) presumably represents the glycosylated form and the 250 $\mathrm{kDa}$ protein (hashes) the deglycosylated form of the protein. Thus, the size of the $N$-glycan moieties was estimated to approximately $40 \mathrm{kDa}$.

MALDI-TOF/TOF analysis of PNGase A/F released, permethylated $N$-glycans from purified HLGP provided the information on their masses and composition. The MS spectra showed presence of high-mannose structures $\mathrm{HexNAc}_{2} \mathrm{Hex}_{9}$ and $\mathrm{HexNAc}_{2} \mathrm{Hex}_{10}$ (ion $\mathrm{m} / \mathrm{z}$ 2396.7 and 2600.8 , respectively) and complex glycans $\mathrm{HexNAc}_{4} \mathrm{Hex}_{7}$ and $\mathrm{HexNAc}_{4} \mathrm{Hex}_{8}(\mathrm{~m} / \mathrm{z} 2477.8$ and 2684, respectively) (Figure 4A). Composition of the glycan structures was confirmed by MS/MS analysis (data not shown). Spectra contained also contaminating series of hexose ions and of $O$-glycans (e.g. ion $\mathrm{m} / \mathrm{z} 1707$, not marked in the figure), present also in other tick samples
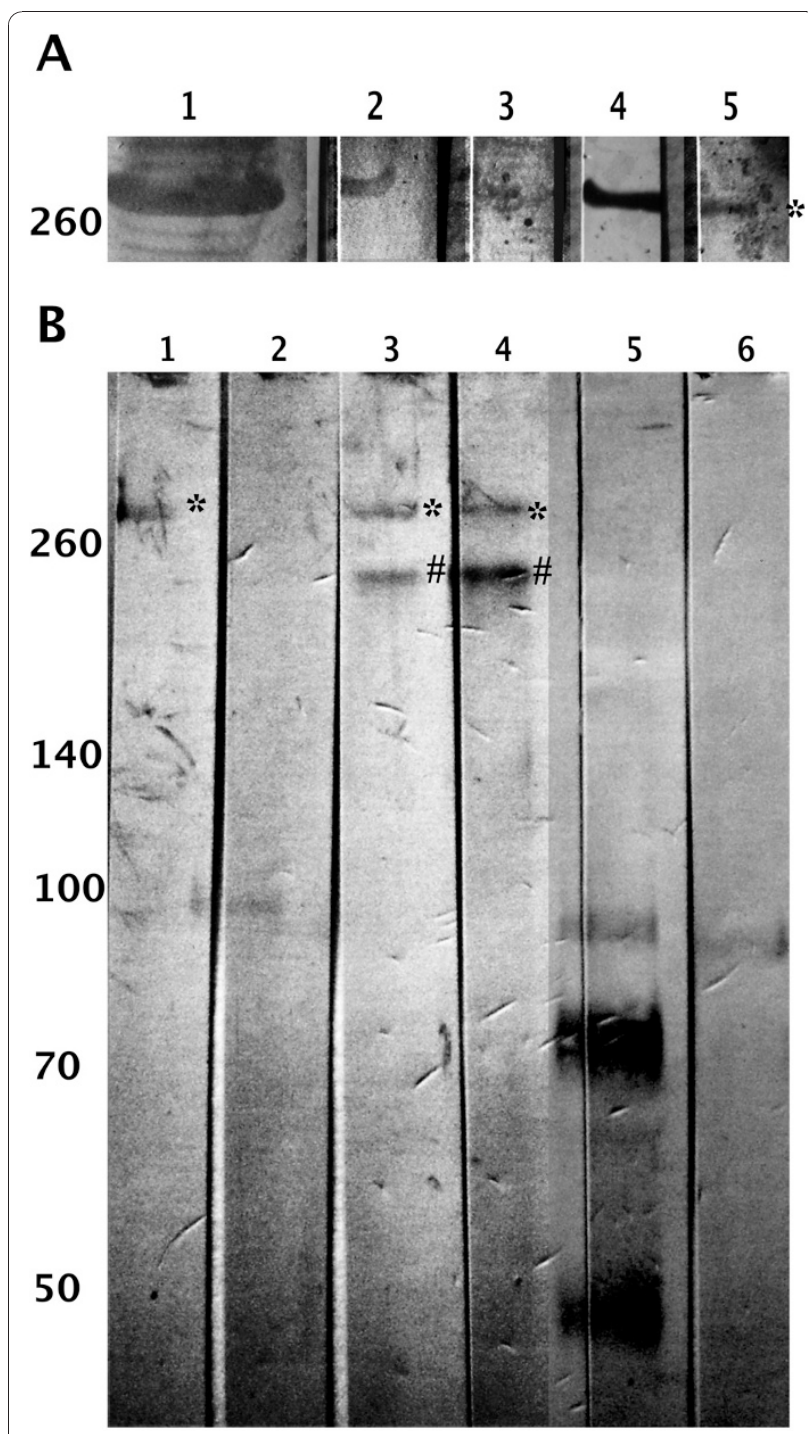

Figure 3 HLGP is a glycosylated protein. Molecular weights of standard proteins are depicted. A) Schiff staining and lectin staining of electrophoretically separated and electroblotted purified HLGP. 1 - tick plasma proteins, stained with Coomassie Brilliant Blue, 2-5 electroblotted HLGP staining. 2 - Schiff staining, 3 - SNA lectin staining, 4 - GNA lectin staining, 5 - DSA lectin staining of purified HLGP. Positive reaction in Schiff staining confirms the presence of glycans on HLGP. Types of glycans identified by positive reactions in lectinoblotting are described in text. B) Immunodetection of deglycosylated purified HLGP using anti-HLGP serum. 1,3,4 - nonreduced electroeluted HLGP and 2,5,6 - reduced electroeluted HLGP stained with anti-HLGP serum. Samples in lanes 3 and 5 were deglycosylated using Endo $\mathrm{H}$, samples in lanes 4 and 6 deglycosylated using PNGase A/F. * - native glycosylated HLGP, \# deglycosylated form of HLGP.

such as salivary glands, gut, hemolymph, ovaria, or whole tick homogenates (unpublished results). For comparison, $\mathrm{N}$-glycans from $D$. marginatus hemolymph were also analyzed. All four glycan structures, identified in HLGP samples, were present also in hemolymph 


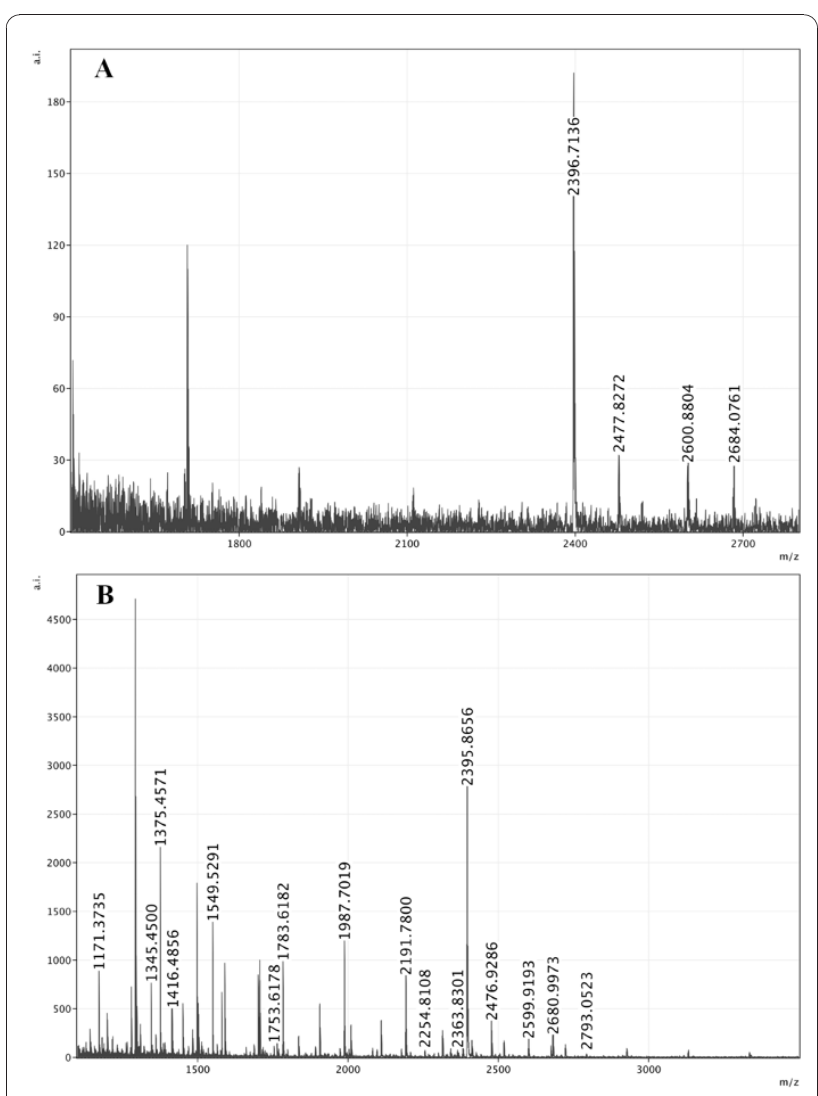

Figure 4 Mass spectra of $\mathrm{N}$-glycans from the purified HLGP and from $D$. marginatus hemolymph. A) MALDI-TOF spectrum of enzymatically released $\mathrm{N}$-glycans from HLGP. Four peaks corresponding to $s \mathrm{HexNAC}_{2} \mathrm{Hex}_{9}$ (m/z 2396.7), $\operatorname{HexNAc}_{2} \mathrm{Hex}_{10}(\mathrm{~m} / \mathrm{z}$ 2600.8), $\mathrm{HexNAc}_{4} \mathrm{Hex}_{7}$ (m/z 2477.8), and HexNAc${ }_{4} \mathrm{Hex}_{8}$ ( $\mathrm{m} / \mathrm{z}$ 2684) are marked. Three more peaks are visible corresponding to contaminating series of hexoses and O-glycans (not labeled). B) MALDI-TOF spectrum of enzymatically released $\mathrm{N}$-glycans from $D$. marginatus hemolymph. A range of glycan structures was identified, namely high-mannose type glycans $\mathrm{HexNAc}_{2} \mathrm{Hex}_{3}$ to $\mathrm{HexNAc}_{2} \mathrm{Hex}_{10}$ ( $\mathrm{m} / \mathrm{z}$ 1171.3, 1375.4, 1783.6, 1987.7, 2191.8, 2395.9, 2599.9) and complex fucosylated and non-fucosylated glycans such as $\mathrm{HexNac}_{3} \mathrm{Hex}_{3}$ (m/z 1416.5), $\mathrm{HexNAc}_{2} \mathrm{Hex}_{4} \mathrm{dHex}_{1}$ (m/z 1549.5), $\mathrm{HexNAc}_{2} \mathrm{Hex}_{5} \mathrm{dHex}_{1}$ (m/z 1753.6). Again, contaminants were present in the sample - series of hexoses as well as O-glycans (not labeled).

(Figure 4B). Other hemolymph protein $N$-glycans were identified as high-mannose type ranging from HexNA$\mathrm{c}_{2} \mathrm{Hex}_{3}$ to $\mathrm{HexNAc}_{2} \mathrm{Hex}_{8}$ (m/z 1171.3, 1375.4, 1783.6, 1987.7, 2191.8) and fucosylated and non-fucosylated complex type glycans such as $\mathrm{HexNac}_{3} \mathrm{Hex}_{3}$ (1416.5), $\mathrm{HexNAc}_{2} \mathrm{Hex}_{4} \mathrm{dHex}_{1}$ (1549.5), HexNAc $\mathrm{Hex}_{5} \mathrm{dHex}_{1}$ (1753.6). Also in this case, a series of hexoses as well as $O$-glycans were present in the sample (Figure 4B).

In accordance with the presence of hemagglutination activity (HA) in hemolymph (unpublished results), the purified HLGP demonstrated HA (Figure 5A). The inhibition of HA with monosaccharides was not performed due to low amounts of sample available and thus the

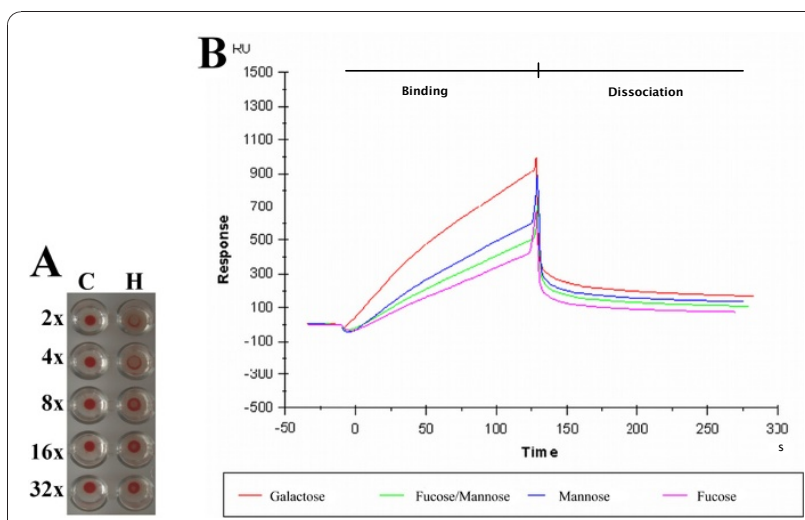

Figure 5 HLGP is a carbohydrate-binding protein - HA and SPR A) Hemagglutination activity of purified HLGP. C - negative control (no hemagglutination activity), $\mathrm{H}$ - serial two-fold dilution of electroeluted HLGP. Hemagglutination activity is visible in dilutions $2 \times$ to $16 \times$. B) Carbohydrate-binding specificity of purified HLGP assessed by SPR on a chip with four channels arranged sequentially as galactose-fucose/mannose-mannose-fucose. Red line corresponds to signal from the channel with immobilized galactose, green to channel with immobilized fucose and mannose, blue to channel with immobilized mannose and purple to channel with immobilized fucose. The fucose/mannose channel contained half amount of the corresponding carbohydrates and serves as control. Lines correspond to SPR response in real-time (not normalized for the sequential flow through channels). Galactose showed the strongest binding by HLGP, some binding was observed also for mannose. Fucose was not recognized by the protein.

carbohydrate specificity was addressed by surface plasmon resonance experiments. A chip with channels with immobilized galactose, fucose, and mannose was used. The channel with immobilized fucose and mannose (half amounts) was used as a control of bindingresponse. The experiment showed binding specificity of the purified HLGP towards galactose (Figure 5B). HLGP showed also a weak binding-specificity towards mannose as seen from comparison of the signal from the mannose channel and the control fucose/mannose channel (Figure 5B). The binding of HLGP to carbohydrates was inhibited by EDTA (data not shown).

\section{Tissue distribution}

The presence of HLGP in tick tissues was determined by immunoblotting. In addition to plasma, hemocytes (as the cellular component of hemolymph) from both fed and unfed females and males, fed female salivary glands (to investigate the presence of HLGP in salivary glands and possibly also in saliva), and gut were investigated.

In all samples, anti-HLGP serum detected the same protein with the same molecular weight (Figure 1B). While amount of the protein was similar in both females and males, regardless of blood-feeding, HGLP was present in higher amounts only in hemocytes from 
fed female tick. In males, the amount of HLGP did not increase during the blood-feeding.

Interestingly, in the salivary glands (Figure 1B, lane 10), the anti-HLGP serum detected two forms of this protein, corresponding in size to the glycosylated (asterisk) and the deglycosylated (hash) forms (compare with Figure 3B, lanes 3,4).

\section{Discussion}

This work was aimed towards the characterization of hemelipoglycoprotein, the most abundant protein of tick hemolymph. To date, only one function of the protein has been identified - the binding and storage of heme $[2,4]$. Surprisingly, in our work, the protein was recognized by antibodies, which were directed against the first isolated tick lectin Dorin M [8] but also sera against the hemagglutination activity of the hemolymph of ticks $D$. marginatus and I. ricinus. Hemagglutination activity (HA) is an ability of some proteins to bind carbohydrates on red blood cells and thus to form clusters. HA is a typical characteristic of lectins, proteins with the ability of specific recognition of specific carbohydrates and their reversible binding. Antibodies raised against HA of tick hemolymph recognize proteins which bind red blood cells in this manner and potentially also proteins, which form tight complexes with them. AntiDorin $\mathrm{M}$ antibodies, on the other hand, react more specifically with proteins containing FReD domain or lectins (unpublished results).

All the used antibodies detected similar proteins in hemolymph; one of the putative lectin molecules with the molecular weight of $290 \mathrm{kDa}$ (with two subunits of 86 and $90 \mathrm{kDa}$ ) was identified as hemelipoglycoprotein (HLGP). The theoretical masses of the subunits for the HLGP from D. variabilis, based on convertase cleavage, are 85 and $93 \mathrm{kDa}$ for HLGP precursor (GenBank accession No. EEC1882) and 79 and $92 \mathrm{kDa}$ for HLGP precursor 2 (GenBank accession No. EEC17915) [16] which clearly corresponds with our results for $D$. marginatus protein. The molecular weight of the protein is similar to other tick hemelipoglycoproteins or carrier proteins (another name of the protein) whose size ranges from $200 \mathrm{kDa}$ to $365 \mathrm{kDa}[2,4]$. The proteins, described by these authors, contained two subunits of 92 and 98 or 92 and $103 \mathrm{kDa}$.

Hemelipoglycoproteins contain the N-terminal lipoprotein domain, a domain of unknown function, and the von Willebrand domain type $\mathrm{D}$, but they do not contain the fibrinogen-related domain, nor any other known lectin domain; moreover, hemelipoglycoproteins are not phylogenetically related to FReD proteins [17]. On the other hand, the $\mathrm{N}$-terminal lipoprotein domain is proposed to have carbohydrate, lipid, and metal-binding properties [16]. The $\mathrm{N}$-terminal lipoprotein domain shares high similarity with vitellogenins from insects and other arthropods and the carbohydrate-binding specificity of vitellogenin was showed for example in the Colorado beetle hemolymph (Leptinotarsa decemlineata) [18]. The lipoprotein domain of the DvCP from D. variabilis is similar also to vitellogenin from fish of the genus Branchiostoma, which also shows hemagglutination activity and carbohydrate-binding ability [19].

HLGP and the other proteins recognized by the antiHA antibodies in the $D$. marginatus hemolymph form a high-molecular weight complex under native conditions (Figure 2A, B). Thus, in the process of anti-HA antibodies preparation, HLGP could be co-purified with proteins with lectin activity. This can explain the anti-HA antibodies binding to HLGP; however, this fact does not explain the binding of anti-Dorin $M$ antibodies to the protein. The structure of HLGP, FReD-containing proteins, and Dorin $M$ are not known and HLGP does not show any similarity to FreD proteins on the primary structure level. However, recognition of HLGP by anti$\mathrm{HA}$ and anti-Dorin $\mathrm{M}$ antibodies, together with carbohydrate-binding SPR studies suggest, that HLGP shares similar epitopes with the lectin domains of the FReDcontaining proteins and that HLGP is structurally, rather than sequentially, similar to lectins/FReD-containing proteins.

The electroeluted and native purified HLGP were able to bind to immobilized monosaccharides in SPR experiments or to hemagglutinate red blood cells (Figure 5A, B). This corresponds to hemagglutination experiments performed on hemolymph from $D$. marginatus (unpublished results) as well as from other ticks $[8,17,20]$. The diminished carbohydrate-binding activity in the presence of EDTA (data not shown) suggests dependency on calcium (divalent) cations, which corresponds to calcium dependence of HA of crayfish plasma vitellogeninrelated protein [21] and sea urchin vitellogenin [22].

FReD-containing proteins are present in vertebrates and in invertebrates. They were described as humoral factors of innate immunity which are able to recognize PAMPs (pathogen-associated molecular patterns); their other functions include participation in regulation of embryo development or in cell adhesion. Donohue and colleagues [16] and Maya-Monteiro and colleagues [2] suggest also other possible roles of carrier proteins in the ticks apart from the heme-storage and prevention of oxidative stress. Our finding of HLGP participation in a high-molecular weight complex with other putative FReD-containing proteins, the presence of two forms of HLGP in tick salivary glands, and its carbohydrate-binding properties point to some other, yet undiscovered, roles of the protein.

HLGP from $D$. marginatus is modified by $N$-glycans as was showed by Schiff staining and lectin affinity staining 
(Figure 3A). PNGase A/F and Endo $\mathrm{H}$ endoglycosidases decreased the size of HLGP by $40 \mathrm{kDa}$ under native conditions (Figure 3B). The reactivity of HLGP with lectins DSA, GNA, and SNA indicated the presence of $N$ glycans with terminal mannose, terminal $\alpha(1-4)$-linked galactose and $\alpha$ (2-6)-linked sialic acid. On the other hand, the absence of binding by the MAA II and PNA lectins suggested the absence of $\alpha(2-3)$-linked sialic acid and of terminal $\alpha(1-3)$-linked galactose. The subsequent mass spectrometric analysis of permethylated $N$-glycans from the purified HLGP revealed the presence of four different glycan moieties. Two were high-mannose type containing two $\mathrm{N}$-acetylhexosamines and nine and ten hexose molecules, and the other two were complex glycans comprising four HexNAcs and seven and eight hexoses, possibly with terminal galactoses. Thus, the mass spectrometric analysis confirmed the results from lectin-affinity staining. Similar glycans were found also in other tick glycoprotein with identified glycans, Dorin $M$ from Ornithodoros moubata [5]. In Dorin M, two high-mannose glycans and a core-fucosylated structure were identified.

Surprisingly, core-fucosylated $N$-glycans were not found in HLGP, even though their presence was confirmed in D. marginatus hemolymph (Figure 4B) and they are the most abundant type of glycans found in tick saliva and salivary glands (Ixodes ricinus, unpublished results). The role of core-fucosylation and protein-specific fucosylation in ticks will be one of the exciting problems of tick glycobiology and physiology.

HLGP has been detected in several tissues of the tick hemolymph, hemocytes, gut, and the protein in both glycosylated and non-glycosylated form also in salivary glands. Previously, HLGP was immunolocalized inside the gut cells, on their surface, as well as inside the salivary duct and epithelium cells of salivary glands (unpublished results). Its presence was shown in these tissues also in other ticks and in different life stages of ticks $[3,4]$. Furthermore, HLGP is present in salivary glands of $D$. marginatus in two forms, one of them corresponding in size to the non-glycosylated form of the hemolymph (glycosylated) protein, the other to the hemolymph protein. While Gudderra and colleagues [3] showed, that DvCP in the salivary glands of D. variabilis does not contain heme in its molecule, the presence of two forms of the protein was not described to date elsewhere. The role of HLGP in tick saliva is not clear nor is the importance of the glycosylation of HLGP or the other tick salivary proteins for the tick feeding.

\section{Conclusion}

A heme-carrier protein from the hemolymph of the ornate sheep tick Dermacentor marginatus, hemelipoglycoprotein, was characterized. The protein is $N$ - glycosylated, bearing high-mannose and complex type glycans without core-fucose. Nevertheless, core-fucosylated glycans are abundant in hemolymph. Hemelipoglycoprotein forms a complex with fibrinogen-related proteins in hemolymph. It shows carbohydrate-binding activity, which is divalent cations-dependent. Moreover, the protein is present in two forms in fed female salivary glands and one of these forms corresponds to the deglycosylated protein. Altogether, these findings suggest also other roles of the protein in ticks different from its heme-binding function. Due to the carbohydrate-binding activity, one of the possibilities is the involvement of hemelipoglycoprotein in innate immunity processes.

\section{Additional material}

Additional File 1: Tryptic fragments identified in

hemelipoglycoprotein precursor [Dermacentor variabilis], coverage 10.2\%. Mass:178646, Score:12286, Queries matched: 244.

Additional File 2: Tryptic fragments identified in hemelipoglycoprotein precursor 2 [Dermacentor variabilis], coverage 4\%. Mass:171972, Score: 707, Queries matched: 12.

Additional File 3: Purification of HLGP. Molecular weights of standard proteins are depicted. A) Immunoprecipitation of HLGP from D. marginatus hemolymph. 1 - Immunostaining of non-reduced HLGP in tick plasma, 2,3 - immunostaining of immunoprecipitated non-reduced HLGP. B) Electroelution of electrophoretically separated HLGP from D. marginatus plasma. SDS-PAGE separated proteins stained with Coomassie Brilliant Blue. 1 - non-reduced plasma proteins, 2 - non-reduced electroeluted HLGP. * marks the position of native glycosylated HLGP, \# marks the deglycosylated form of HLGP.

\section{Abbreviations}

AP: alkaline phosphatase; CP: carrier proteins; dHex: deoxyhexose; DSA: Datura stramonium agglutinin; FReD: fibrinogen-related domain; FReP: fibrinogen-related protein; GNA: Galanthus nivalis agglutinin; HA: hemagglutination activity; Hex: hexose; HexNAc: N-acetyl hexosamine; HLGP: hemelipoglycoprotein; MAA: Maackia amurensis agglutinin; PAMP: pathogenassociated molecular pattern; SNA: Sambucus nigra agglutinin; SPR: surface plasmon resonance.

\section{Acknowledgements}

This work was supported by Ministry of Education, Youth and Sports of the Czech Republic (6007665801, LC06009), Grant Agency of the Academy of Sciences of Czech Republic (KJB600960906), and the Student Grant Agency of the University of South Bohemia (SGA2008/017, SGA2009/017). We thank Milos V. Novotny, Benjamin F. Mann, and William R. Alley, Jr. for the MS analysis, Michaela Wimmerova and Lenka Malinovska for the SPR analysis, Jan Lopatar for help with manuscript preparation, Jan Erhart for the work with ticks, Lenka Bucinska for tick dissection, and Lenka Stifterova for help with hemagglutination assays and immune sera preparation.

\section{Author details}

${ }^{1}$ Faculty of Science, University of South Bohemia, Branisovska 31, CZ-37005 Ceske Budejovice, Czech Republic. ${ }^{2}$ Institute of Parasitology, Biology Centre of the Academy of Sciences of the Czech Republic, Branisovska 31, CZ-37005 Ceske Budejovice, Czech Republic.

\section{Authors' contributions}

JD conducted electrophoreses, blotting experiments, HA assay, and participated in SPR experiments, JD and JS participated in anti-HLGP and anti-tick HA sera preparation and in HLGP purification, JS and MV performed mass spectrometry and analysis of data, MV performed tick tissue 
preparation and participated in their analysis, LG co-ordinated the experiments, all authors participated in the design of experiments, manuscript preparation, and they approved the final manuscript.

\section{Competing interests}

The authors declare that they have no competing interests.

Received: 15 October 2010 Accepted: 7 January 2011

Published: 7 January 2011

\section{References}

1. Braz GRC, Coelho HSL, Masuda H, Oliveira PL: A missing metabolic pathway in the cattle tick Boophilus microplus. Curr Biol 1999, 9:703-706.

2. Maya-Monteiro CM, Daffre S, Logullo C, Lara FA, Alves EW, Capurro ML, Zingali R, Almeida IC, Oliveira PL: HeLp, a heme lipoprotein from the hemolymph of the cattle tick, Boophilus microplus. J Biol Chem 2000, 275:36584-36589.

3. Gudderra NP, Sonenshine DE, Apperson CS, Roe RM: Tissue distribution and characterization of predominant hemolymph carrier proteins from Dermacentor variabilis and Ornithodoros parkeri. I Insect Physiol 2002, 48:161-170.

4. Gudderra NP, Neese PA, Sonenshine DE, Apperson CS, Roe RM: Developmental profile, isolation, and biochemical characterization of a novel lipoglycoheme-carrier protein from the American dog tick, Dermacentor variabilis (Acari: Ixodidae) and observation on a similar protein in the soft tick, Ornithodoros parkeri (Acari: Argasidae). Insect Biochem Mol Biol 2001, 31:299-311.

5. Man P, Kováŕ V, Štěrba J, Strohalm M, Kavan D, Kopáček P, Grubhoffer L, Havliček V: Deciphering Dorin M glycosylation by mass spectrometry. Eur J Mass Spectrom 2008, 14:345-354.

6. Gudderra NP, Sonenshine DE, Apperson CS, Roe RM: Hemolymph proteins in ticks. J Insect Physiol 2002, 48:269-278.

7. Maya-Monteiro CM, Alves LR, Pinhal N, Abdalla DSP, Oliveira PL: HeLp, a heme-transporting lipoprotein with an antioxidant role. Insect Biochem Mol Biol 2004, 34:81-87.

8. Kovár $V$, Kopáček P, Grubhoffer L: Isolation and characterization of Dorin $\mathrm{M}$, a lectin from plasma of the soft tick Ornithodoros moubata. Insect Biochem Mol Biol 2000, 30:195-205.

9. Laemmli UK: Cleavage of structural proteins during the assembly of the head of bacteriophage T4. Nature 1970, 227:680-685.

10. Wittig I, Braun HP, Schägger H: Blue native PAGE. Nat Protoc 2006, 1:418-428.

11. Grubhoffer $L$, Kováŕ $V$ : Arthropod lectins: affinity approaches in the analysis and preparation of carbohydrate binding proteins. In Techniques in Insect Immunology FITC-5. Edited by: Wiesner A, Dunphy GG, Marmaras VJ, Morishima I, Sugumaran M, Yamakawa M. Fair Haven, New Jersey: SOS Publications; 1998:47-57.

12. Towbin $\mathrm{H}$, Staehlin T, Gordon J: Electrophoretic transfer of proteins from polyacrylamide gels to nitrocellulose sheets: procedure and some applications. Proc Nat Acad Sci USA 1979, 76:4350-4354.

13. Alley WR Jr, Madera M, Mechref Y, Novotny MV: Chip-based reversedphase liquid chromatography-mass spectrometry of permethylated $\mathrm{N}$ linked glycans: A potential methodology for cancer-biomarker discovery. Anal Chem 2010, 82:5095-5106.

14. Strohalm $M$, Hassman $M$, Košata $B$, Kodíček $M$ : mMass data miner: an open source alternative for mass spectrometric data analysis. Rapid Commum Mass Spectrom 2008, 22:905-9.

15. Ceroni A, Maass K, Geyer H, Geyer R, Dell A, Haslam SM: GlycoWorkbench: A tool for the computer-assisted annotation of mass spectra of glycans. J Prot Res 2008, 7:1650-1659.

16. Donohue KV, Khalil SM, Mitchell RD, Sonenshine DE, Roe RM: Molecular characterization of the major hemelipoglycoprotein in ixodid ticks. Insect Mol Biol 2008, 17:197-208.

17. Sterba J, Dupejova J, Fiser M, Golovchenko M, Rudenko N, Grubhoffer L: Identification and characterization of lectins in several tick species [abstract]. FEBS J 2009, 276:88.

18. Stynen D, De Loof A: Sugar binding properties of the vitellogenic proteins in the Colorado beetle, demonstrated by hemagglutination and precipitation experiments. Rouxs Arch Dev Biol 1982, 191:159-162.

19. Zhang S, Sun Y, Pang Q, Shi X: Hemagglutinating and antibacterial activities of vitellogenin. Fish \& Shellfish Immun 2005, 19:93-95.
20. Kibuka-Sebitosi E: Potential role of lectins in ticks: Rhipicephalus appendiculatus and Rhipicephalus pulchellus (Acari: Ixodidae). Syst Appl Acarol 2006, 21:1-15.

21. Hall $M$, Wang $R$, van Antwerpen $R$, Sottrup-Jensen $L$, Söderhäll $K$ : The crayfish plasma clotting protein: A vitellogenin-related protein responsible for clot formation in crustacean blood. Proc Nat Acad Sci USA 1999, 965:1965-1970.

22. Cervello M, Arizza V, Cammarata M, Matranga V, Parrinelo N: Properties of sea urchin coelomocyte agglutinin. Ital J Zool 1996, 63:353-356.

doi:10.1186/1756-3305-4-4

Cite this article as: Dupejova et al.: Hemelipoglycoprotein from the ornate sheep tick, Dermacentor marginatus: structural and functional characterization. Parasites \& Vectors 2011 4:4.

\section{Submit your next manuscript to BioMed Central and take full advantage of:}

- Convenient online submission

- Thorough peer review

- No space constraints or color figure charges

- Immediate publication on acceptance

- Inclusion in PubMed, CAS, Scopus and Google Scholar

- Research which is freely available for redistribution

Submit your manuscript at www.biomedcentral.com/submit
C Biomed Central 